\title{
The Capacity Region of a Class of Discrete Degraded Interference Channels
}

\author{
Nan Liu and Sennur Ulukus
}

\begin{abstract}
We provide a single-letter characterization for the capacity region of a class of discrete degraded interference channels (DDICs). The class of DDICs considered includes the discrete additive degraded interference channel (DADIC) studied by Benzel [1]. We show that for the class of DDICs studied, encoder cooperation does not increase the capacity region, and therefore, the capacity region of the class of DDICs is the same as the capacity region of the corresponding degraded broadcast channel.
\end{abstract}

\section{INTRODUCTION}

In wireless communications, where multiple transmitter and receiver pairs share the same medium, interference is unavoidable. How to best manage interference coming from other users and how not to cause too much interference to other users while maintaining the quality of communication is a challenging question and of a great deal of practical interest.

To be able to understand the effect of interference on communications better, interference channel (IC) has been introduced in [2]. The IC is a simple network consisting of two pairs of transmitters and receivers. Each pair wishes to communicate at a certain rate with negligible probability of error. However, the two communications interfere with each other. To best understand the management of interference, we need to find the capacity region of the IC. However, the problem of finding the capacity region of the IC is essentially open except in some special cases, e.g., a class of deterministic ICs [3], discrete additive degraded interference channels (DADICs) [1], strong ICs [4], [5], ICs with statistically equivalent outputs [6]-[8].

In this paper, we consider a class of discrete degraded interference channels (DDICs). In a DDIC, only the "bad" receiver faces interference, while the "good" receiver has the ability to decode both messages and thus, behaves like the receiver of a multiple access channel. It is this fact that makes the DDIC easier to analyze as compared to the IC, where both receivers are faced with interference.

We provide a single-letter characterization for the capacity region of a class of DDICs. The class of DDICs includes the DADICs studied by Benzel [1]. We show that for the class of DDICs studied, encoder cooperation does not increase the capacity region, and therefore, the capacity region of the class of DDICs is the same as the capacity region of the corresponding degraded broadcast channel, which is known.

This work was supported by NSF Grants CCR 03-11311, CCF 04-47613 and CCF 05-14846.

N. Liu and S. Ulukus are with the Department of Electrical and Computer Engineering, University of Maryland, College Park, MD 20742, USA nkancy@umd.edu; ulukus@umd.edu

\section{SYSTEM MODEL}

A discrete memoryless IC consists of two transmitters and two receivers. Transmitter 1 has message $W_{1}$ to send to receiver 1 . Transmitter 2 has message $W_{2}$ to send to receiver 2. Messages $W_{1}$ and $W_{2}$ are independent. The channel consists of two input alphabets, $\mathcal{X}_{1}$ and $\mathcal{X}_{2}$, and two output alphabets, $\mathcal{Y}_{1}$ and $\mathcal{Y}_{2}$. The channel transition probability is $p\left(y_{1}, y_{2} \mid x_{1}, x_{2}\right)$.

In this paper, our definition of degradedness is in the stochastic sense, i.e., we say that an IC is DDIC if there exists a probability distribution $p^{\prime}\left(y_{2} \mid y_{1}\right)$ such that

$$
p\left(y_{2} \mid x_{1}, x_{2}\right)=\sum_{y_{1} \in \mathcal{Y}_{1}} p\left(y_{1} \mid x_{1}, x_{2}\right) p^{\prime}\left(y_{2} \mid y_{1}\right)
$$

for all $x_{1} \in \mathcal{X}_{1}, x_{2} \in \mathcal{X}_{2}$ and $y_{2} \in \mathcal{Y}_{2}$. However, we note that for any DDIC, we can form another DDIC (physically degraded) by

$$
p\left(y_{1}, y_{2} \mid x_{1}, x_{2}\right)=p\left(y_{1} \mid x_{1}, x_{2}\right) p^{\prime}\left(y_{2} \mid y_{1}\right)
$$

which has the same marginals, $p\left(y_{1} \mid x_{1}, x_{2}\right)$ and $p\left(y_{2} \mid x_{1}, x_{2}\right)$, as the original DDIC. Since the receivers do not cooperate in an IC, similar to the case of the broadcast channel [9, Problem 14.10], the capacity region is only a function of the marginals, $p\left(y_{1} \mid x_{1}, x_{2}\right)$ and $p\left(y_{2} \mid x_{1}, x_{2}\right)$, and the rate pairs in the capacity region can be achieved by the same achievability scheme for different ICs with the same marginals. Hence, the capacity results that we obtain for DDICs which satisfy (2) will be valid for any DDIC that has the same marginals, $p\left(y_{1} \mid x_{1}, x_{2}\right)$ and $p\left(y_{2} \mid x_{1}, x_{2}\right)$. Thus, without loss of generality, from now on, we may restrict ourselves to studying DDICs that satisfy (2).

A DDIC is characterized by two transition probabilities, $p^{\prime}\left(y_{2} \mid y_{1}\right)$ and $p\left(y_{1} \mid x_{1}, x_{2}\right)$. For notational convenience, let $T^{\prime}$ denote the $\left|\mathcal{Y}_{2}\right| \times\left|\mathcal{Y}_{1}\right|$ matrix of transition probabilities $p^{\prime}\left(y_{2} \mid y_{1}\right)$, and $T_{\bar{x}_{2}}$ denote the $\left|\mathcal{Y}_{1}\right| \times\left|\mathcal{X}_{1}\right|$ matrix of transition probabilities $p\left(y_{1} \mid x_{1}, \bar{x}_{2}\right)$, for all $\bar{x}_{2} \in \mathcal{X}_{2}$.

Throughout the paper, $\Delta_{n}$ will denote the probability simplex

$$
\left\{\left(p_{1}, p_{2}, \cdots, p_{n}\right) \mid \sum_{i=1}^{n} p_{i}=1, \quad p_{i} \geq 0, i=1,2, \cdots, n\right\}
$$

and $\mathcal{J}_{n}$ will denote the representation of the symmetric group of permutations of $n$ objects by the $n \times n$ permutation matrices.

The class of DDICs we consider in this paper satisfies the following conditions: 
1) $T^{\prime}$ is input symmetric. Let the input symmetry group be $\mathcal{G}$.

2) For any $x_{2}^{\prime}, x_{2}^{\prime \prime} \in \mathcal{X}_{2}$, there exists a permutation matrix $G \in \mathcal{G}$, such that

$$
T_{x_{2}^{\prime}}=G T_{x_{2}^{\prime \prime}}
$$

3) $H\left(Y_{1} \mid X_{1}=x_{1}, X_{2}=x_{2}\right)=\eta$, independent of $x_{1}$, $x_{2}$.

4) $p\left(y_{1} \mid x_{1}, x_{2}\right)$ satisfies

$$
\sum_{x_{2}} p\left(y_{1} \mid x_{1}, x_{2}\right)=\frac{\left|\mathcal{X}_{2}\right|}{\left|\mathcal{Y}_{1}\right|}, \quad x_{1} \in \mathcal{X}_{1}, y_{1} \in \mathcal{Y}_{1}
$$

5) Let $\mathbf{p}_{x_{1}, x_{2}}$ be the $\left|\mathcal{Y}_{1}\right|$ dimensional vector of probabilities $p\left(y_{1} \mid x_{1}, x_{2}\right)$ for a given $x_{1}, x_{2}$. Then, there exists an $\tilde{x}_{2} \in \mathcal{X}_{2}$, such that

$$
\begin{aligned}
& \left\{\sum_{x_{1}, x_{2}} a_{x_{1}, x_{2}} \mathbf{p}_{x_{1}, x_{2}}: \sum_{x_{1}, x_{2}} a_{x_{1}, x_{2}}=1, a_{x_{1}, x_{2}} \geq 0\right\} \\
\subseteq & \left\{G\left(\sum_{x_{1}} b_{x_{1}} \mathbf{p}_{x_{1}, \tilde{x}_{2}}\right): \sum_{x_{1}} b_{x_{1}}=1, b_{x_{1}} \geq 0, G \in \mathcal{G}\right\}
\end{aligned}
$$

The definition of an input symmetric channel is given in [10, Section II.D]. For completeness, we repeat it here. For an $m \times n$ stochastic matrix $T^{\prime}$ (an $n$ input, $m$ output channel), the input symmetry group $\mathcal{G}$ is defined as

$$
\mathcal{G}=\left\{G \in \mathcal{J}_{n}: \exists \Pi \in \mathcal{J}_{m}, \quad T^{\prime} G=\Pi T^{\prime}\right\}
$$

i.e., $\mathcal{G}$ is the set of permutation matrices $G$ such that the column permutations of $T^{\prime}$ with $G$ may be achieved with corresponding row permutations. $T^{\prime}$ is input symmetric, if $\mathcal{G}$ is transitive, i.e., any element of $\{1,2, \cdots, n\}$ can be mapped to every other element of $\{1,2, \cdots, n\}$ by some member of $\mathcal{G}$. $\mathcal{G}$ being a transitive subgroup means that the output entropy of channel $T^{\prime}$ is maximized when the input distribution is chosen to be the uniform distribution, i.e.,

$$
\max _{\mathbf{p} \in \Delta_{n}} H\left(T^{\prime} \mathbf{p}\right)=H\left(T^{\prime} \mathbf{u}\right)
$$

where $\mathbf{u}$ denotes the uniform distribution in $\Delta_{n}$. This is because, for any $\mathbf{p} \in \Delta_{n}$, if we let $\mathbf{q}=|\mathcal{G}|^{-1} \sum_{G \in \mathcal{G}} G \mathbf{p}$, then we have

$$
\begin{aligned}
H\left(T^{\prime} \mathbf{q}\right) & =H\left(|\mathcal{G}|^{-1} \sum_{G \in \mathcal{G}} T^{\prime} G \mathbf{p}\right) \\
& =H\left(|\mathcal{G}|^{-1} \sum_{G \in \mathcal{G}} \Pi_{G} T^{\prime} \mathbf{p}\right) \\
& \geq|\mathcal{G}|^{-1} \sum_{G \in \mathcal{G}} H\left(\Pi_{G} T^{\prime} \mathbf{p}\right) \\
& =H\left(T^{\prime} \mathbf{p}\right)
\end{aligned}
$$

where (10) follows from the fact that $G \in \mathcal{G}$, and 111 follows from the concavity of the entropy function. Note that for any $G^{\prime} \in \mathcal{G}$,

$$
G^{\prime} \mathbf{q}=\mathbf{q}
$$

by the fact that $\mathcal{G}$ is a group. Since $\mathcal{G}$ is also transitive, $\mathbf{q}=\mathbf{u}$.

Condition 2 implies that for any $p\left(x_{1}\right), H\left(Y_{1} \mid X_{2}=\right.$ $x_{2}$ ) does not depend on $x_{2}$. Combined with condition 1 , condition 2 further implies that $H\left(Y_{2} \mid X_{2}=x_{2}\right)$ does not depend on $x_{2}$ either. These two facts will be proved and utilized in other proofs later.

A sufficient condition for condition 3 to hold is that the vectors $p\left(y_{1} \mid X_{1}=x_{1}, X_{2}=x_{2}\right)$ for all $\left(x_{1}, x_{2}\right) \in \mathcal{X}_{1} \times \mathcal{X}_{2}$ are permutations of each other. This is true for instance when the channel from $Y_{1}$ to $Y_{2}$ is additive [1].

By condition 4, we can show that when $X_{2}$ takes the uniform distribution, $Y_{1}$ will also be uniformly distributed. Combined with condition 1 , condition 4 implies that when $X_{2}$ takes the uniform distribution, $H\left(Y_{2}\right)$ is maximized, irrespective of $p\left(x_{1}\right)$.

In condition 5 , the first line of (6) denotes the set of all convex combinations of vectors $\mathbf{p}_{x_{1}, x_{2}}$ for all $x_{1}, x_{2} \in \mathcal{X}_{1} \times$ $\mathcal{X}_{2}$, while the second line denotes all convex combinations, and their permutations with $G \in \mathcal{G}$, of vectors $\mathbf{p}_{x_{1}, \tilde{x}_{2}}$ for all $x_{1} \in \mathcal{X}_{1}$, but for a fixed $\tilde{x}_{2} \in \mathcal{X}_{2}$. Therefore, this condition means that all convex combinations of $\mathbf{p}_{x_{1}, x_{2}}$ may be obtained by a combination of convex combinations of $\mathbf{p}_{x_{1}, \tilde{x}_{2}}$ for a fixed $\tilde{x}_{2}$, and permutations in $\mathcal{G}$.

The DADICs considered in [1] satisfy conditions $1-5$, as we will show in Section VI-A

The aim of this paper is to provide a single-letter characterization for the capacity region of DDICs that satisfy conditions 1-5, and we will follow the proof technique of [1] with appropriate generalizations.

\section{The OUter Bound (CONVERSE)}

When we assume that the encoders are able to fully cooperate, i.e., both encoders know both messages $W_{1}$ and $W_{2}$, we get a corresponding degraded broadcast channel with input $x=\left(x_{1}, x_{2}\right)$. The capacity region of the corresponding degraded broadcast channel serves as an outer bound on the capacity region of the DDIC. The capacity region of the degraded broadcast channel is known [9], [11], [12], and thus, a single-letter outer bound on the capacity region of the DDIC is

$$
\begin{aligned}
& \overline{\mathrm{co}}\left[\bigcup _ { p ( u ) , p ( x _ { 1 } , x _ { 2 } | u ) } \left\{\left(R_{1}, R_{2}\right): R_{1} \leq I\left(X_{1}, X_{2} ; Y_{1} \mid U\right)\right.\right. \\
& \left.\left.R_{2} \leq I\left(U ; Y_{2}\right)\right\}\right]
\end{aligned}
$$

where $\overline{\mathrm{co}}$ denotes the closure of the convex hull operation, and the auxiliary random variable $U$, which satisfies the Markov chain $U \longrightarrow\left(X_{1}, X_{2}\right) \longrightarrow Y_{1} \longrightarrow Y_{2}$, has cardinality bounded by $|\mathcal{U}| \leq \min \left(\left|\mathcal{Y}_{1}\right|,\left|\mathcal{Y}_{2}\right|,\left|\mathcal{X}_{1}\right|\left|\mathcal{X}_{2}\right|\right)$. More specifically, for DDICs that satisfy condition 3, 14 
can be written as

$$
\begin{aligned}
& \overline{\mathrm{co}}\left[\bigcup _ { p ( u ) , p ( x _ { 1 } , x _ { 2 } | u ) } \left\{\left(R_{1}, R_{2}\right): R_{1} \leq H\left(Y_{1} \mid U\right)-\eta\right.\right. \\
& \left.\left.R_{2} \leq I\left(U ; Y_{2}\right)\right\}\right]
\end{aligned}
$$

Let us define $T(c)$ as

$$
T(c)=\max _{p(u) p\left(x_{1}, x_{2} \mid u\right)} I\left(U ; Y_{2}\right)
$$

where the entropies are calculated according to the distribution

$$
p\left(u, x_{1}, x_{2}, y_{1}, y_{2}\right)=p(u) p\left(x_{1}, x_{2} \mid u\right) p\left(y_{1} \mid x_{1}, x_{2}\right) p^{\prime}\left(y_{2} \mid y_{1}\right)
$$

Using condition 3, we can show that $\eta \leq c \leq \log \left|\mathcal{Y}_{1}\right| . T(c)$ is concave in $c$ [1], [13], and therefore, 115 can also be written as

$$
\begin{array}{r}
\bigcup_{\eta \leq c \leq \log \left|\mathcal{Y}_{1}\right|}\left\{\left(R_{1}, R_{2}\right):\right. \\
R_{1} \leq c-\eta \\
\left.R_{2} \leq T(c)\right\}
\end{array}
$$

\section{An Achievable Region}

Based on [7, Theorem 4], the following region is achievable,

$$
\begin{aligned}
& \overline{\mathrm{co}}\left[\bigcup _ { p ( x _ { 1 } ) , p ( x _ { 2 } ) } \left\{\left(R_{1}, R_{2}\right): R_{1} \leq I\left(X_{1} ; Y_{1} \mid X_{2}\right)\right.\right. \\
& \left.\left.R_{2} \leq I\left(X_{2} ; Y_{2}\right)\right\}\right]
\end{aligned}
$$

which corresponds to the achievability scheme that the "bad" receiver treats the signal for the "good" receiver as pure noise, and the "good" receiver decodes both messages as if it is the receiver in a multiple access channel.

For DDICs that satisfy condition 3, (19) reduces to

$$
\begin{aligned}
\overline{\mathrm{co}}\left[\bigcup _ { p ( x _ { 1 } ) , p ( x _ { 2 } ) } \left\{\left(R_{1}, R_{2}\right):\right.\right. & R_{1} \leq H\left(Y_{1} \mid X_{2}\right)-\eta \\
& \left.\left.R_{2} \leq H\left(Y_{2}\right)-H\left(Y_{2} \mid X_{2}\right)\right\}\right]
\end{aligned}
$$

We note that 20 remains an achievable region if we choose $p\left(x_{2}\right)$ to be the uniform distribution. Furthermore, by choosing $p\left(x_{2}\right)$ as the uniform distribution, we have

$$
\begin{aligned}
p\left(y_{1}\right) & =\sum_{x_{1}, x_{2}} p\left(y_{1} \mid x_{1}, x_{2}\right) p\left(x_{1}\right) \frac{1}{\left|\mathcal{X}_{2}\right|} \\
& =\frac{1}{\left|\mathcal{X}_{2}\right|} \sum_{x_{1}} p\left(x_{1}\right) \sum_{x_{2}} p\left(y_{1} \mid x_{1}, x_{2}\right) \\
& =\frac{1}{\left|\mathcal{Y}_{1}\right|}
\end{aligned}
$$

where (23) uses condition 4 . Thus, when $p\left(x_{2}\right)$ is chosen as the uniform distribution, $p\left(y_{1}\right)$ results in a uniform distribution as well. Let us define $\tau$ as

$$
\tau=\max _{\mathbf{p} \in \Delta_{\left|\mathcal{Y}_{1}\right|}} H\left(T^{\prime} \mathbf{p}\right)
$$

Using the fact that the DDIC under consideration satisfies condition 1, i.e., it satisfies (8), we have that when $p\left(x_{2}\right)$ is uniform, and consequently $p\left(y_{1}\right)$ is uniform,

$$
H\left(Y_{2}\right)=\tau
$$

Hence, choosing $p\left(x_{2}\right)$ to be the uniform distribution in (20), yields the following as an achievable region,

$$
\begin{aligned}
\overline{\mathrm{co}}\left[\bigcup _ { p ( x _ { 1 } ) } \left\{\left(R_{1}, R_{2}\right):\right.\right. & R_{1} \leq \frac{1}{\left|\mathcal{X}_{2}\right|} \sum_{x_{2}} H\left(Y_{1} \mid X_{2}=x_{2}\right)-\eta \\
R_{2} & \left.\left.\leq \tau-\frac{1}{\left|\mathcal{X}_{2}\right|} \sum_{x_{2}} H\left(Y_{2} \mid X_{2}=x_{2}\right)\right\}\right]
\end{aligned}
$$

Due to condition 2 , for any $p\left(x_{1}\right)=\mathbf{p}$ and any $x_{2}^{\prime}, x_{2}^{\prime \prime} \in \mathcal{X}_{2}$, there exists a permutation matrix $G \in \mathcal{G}$ such that

$$
\begin{aligned}
H\left(Y_{1} \mid X_{2}=x_{2}^{\prime}\right) & =H\left(T_{x_{2}^{\prime}} \mathbf{p}\right) \\
& =H\left(G T_{x_{2}^{\prime \prime}} \mathbf{p}\right) \\
& =H\left(T_{x_{2}^{\prime \prime}} \mathbf{p}\right) \\
& =H\left(Y_{1} \mid X_{2}=x_{2}^{\prime \prime}\right)
\end{aligned}
$$

which means that for any $p\left(x_{1}\right), H\left(Y_{1} \mid X_{2}=x_{2}\right)$ does not depend on $x_{2}$. Furthermore, for any $p\left(x_{1}\right)=\mathbf{p}$ and any $x_{2}^{\prime}, x_{2}^{\prime \prime} \in \mathcal{X}_{2}$, there exist permutation matrices $G \in \mathcal{G}$ and $\Pi$, of order $\left|\mathcal{Y}_{1}\right|$ and $\left|\mathcal{Y}_{2}\right|$ respectively, such that

$$
\begin{aligned}
H\left(Y_{2} \mid X_{2}=x_{2}^{\prime}\right) & =H\left(T^{\prime} T_{x_{2}^{\prime}} \mathbf{p}\right) \\
& =H\left(T^{\prime} G T_{x_{2}^{\prime \prime}} \mathbf{p}\right) \\
& =H\left(\Pi T^{\prime} T_{x_{2}^{\prime \prime}} \mathbf{p}\right) \\
& =H\left(T^{\prime} T_{x_{2}^{\prime \prime}} \mathbf{p}\right) \\
& =H\left(Y_{2} \mid X_{2}=x_{2}^{\prime \prime}\right)
\end{aligned}
$$

where (33) follows from the fact that $G \in \mathcal{G}$. (35) means that for any $p\left(x_{1}\right), H\left(Y_{2} \mid X_{2}=x_{2}\right)$ does not depend on $x_{2}$ either. Hence, the achievable region in 26 can further be written as

$$
\begin{aligned}
& \overline{\mathrm{co}}\left[\bigcup _ { p ( x _ { 1 } ) } \left\{\left(R_{1}, R_{2}\right): R_{1} \leq H\left(Y_{1} \mid X_{2}=x_{2}\right)-\eta\right.\right. \\
& \left.\left.R_{2} \leq \tau-H\left(Y_{2} \mid X_{2}=x_{2}\right)\right\}\right]
\end{aligned}
$$

for any $x_{2} \in \mathcal{X}_{2}$. Since we will use condition 5 later, we choose to write the region of (36) as

$$
\begin{aligned}
& \overline{\mathrm{co}}\left[\bigcup _ { p ( x _ { 1 } ) } \left\{\left(R_{1}, R_{2}\right): R_{1} \leq H\left(Y_{1} \mid X_{2}=\tilde{x}_{2}\right)-\eta\right.\right. \\
& \left.\left.R_{2} \leq \tau-H\left(Y_{2} \mid X_{2}=\tilde{x}_{2}\right)\right\}\right]
\end{aligned}
$$


where $\tilde{x}_{2}$ is given in condition 5 .

Let us define $F(c)$ as

$$
F(c)=\underset{p\left(x_{1}\right)}{H\left(Y_{1} \mid X_{2}=\tilde{x}_{2}\right)=c} \quad H\left(Y_{2} \mid X_{2}=\tilde{x}_{2}\right)
$$

where the entropies are calculated according to the distribution

$$
p\left(y_{1}, y_{2}, x_{1} \mid \tilde{x}_{2}\right)=p\left(x_{1}\right) p\left(y_{1} \mid x_{1}, \tilde{x}_{2}\right) p^{\prime}\left(y_{2} \mid y_{1}\right)
$$

In (38), we can write min instead of inf by the same reasoning as in [14, Section I]. Note that $F(c)$ is not a function of $\tilde{x}_{2}$ because of (30) and (35). Again, by condition 3 , we can show that $\eta \leq c \leq \log \left|\mathcal{Y}_{1}\right|$. Hence, the achievable region in (37) can be written as,

$$
\begin{aligned}
& \overline{\mathrm{co}}\left[\bigcup _ { \eta \leq c \leq \operatorname { l o g } | \mathcal { Y } _ { 1 } | } \left\{\left(R_{1}, R_{2}\right): R_{1} \leq c-\eta\right.\right. \\
& \left.\left.R_{2} \leq \tau-F(c)\right\}\right]
\end{aligned}
$$

which by [1, Facts 4 and 5], can further be written as

$$
\begin{array}{r}
\bigcup_{\eta \leq c \leq \log \left|\mathcal{Y}_{1}\right|}\left\{\left(R_{1}, R_{2}\right):\right. \\
R_{1} \leq c-\eta \\
\left.R_{2} \leq \tau-\underline{\operatorname{env}} F(c)\right\}
\end{array}
$$

where $\underline{\operatorname{env}} F(\cdot)$ denotes the lower convex envelope of the function $F(\cdot)$.

\section{THE CAPACITY REgION}

In this section, we show that the achievable region in (41) contains the outer bound in (18), and thus, (18) and (41) are both, in fact, single-letter characterizations of the capacity region of DDICs satisfying conditions 1-5. To show this, it suffices to prove that

$$
T(c) \leq \tau-\underline{\operatorname{env}} F(c), \quad \eta \leq c \leq \log \left|\mathcal{Y}_{1}\right|
$$

Let us fix a $c \in\left[\eta, \log \left|\mathcal{Y}_{1}\right|\right]$. Let $p^{*}(u), p^{*}\left(x_{1}, x_{2} \mid u\right)$ be the distributions that achieve the maximum in (16), i.e.,

$$
\begin{aligned}
H\left(Y_{1} \mid U\right) & =c \\
I\left(U ; Y_{2}\right) & =T(c)
\end{aligned}
$$

Using condition 5 , for each $u \in \mathcal{U}$, there exists a $p^{u}\left(x_{1}\right)=$ $\mathbf{p}^{u}$ and a permutation matrix $G^{u} \in \mathcal{G}$, such that

$$
\sum_{x_{1}, x_{2}} p^{*}\left(x_{1}, x_{2} \mid U=u\right) \mathbf{p}_{x_{1}, x_{2}}=G^{u} T_{\tilde{x}_{2}} \mathbf{p}^{u}
$$

Thus, we have

$$
H\left(Y_{1} \mid U=u\right)=H\left(G^{u} T_{\tilde{x}_{2}} \mathbf{p}^{u}\right)=H\left(T_{\tilde{x}_{2}} \mathbf{p}^{u}\right)
$$

(46) means that $\mathbf{p}^{u}$ is in the feasible set of the optimization in (38) when $c=H\left(Y_{1} \mid U=u\right)$. Hence,

$$
F\left(H\left(Y_{1} \mid U=u\right)\right) \leq H\left(T^{\prime} T_{\tilde{x}_{2}} \mathbf{p}^{u}\right)
$$

We have

$$
\begin{aligned}
H\left(Y_{2} \mid U=u\right) & =H\left(T^{\prime} G^{u} T_{\tilde{x}_{2}} \mathbf{p}^{u}\right) \\
& =H\left(\Pi^{u} T^{\prime} T_{\tilde{x}_{2}} \mathbf{p}^{u}\right) \\
& =H\left(T^{\prime} T_{\tilde{x}_{2}} \mathbf{p}^{u}\right) \\
& \geq F\left(H\left(Y_{1} \mid U=u\right)\right)
\end{aligned}
$$

where (48, (49) and (51) follow from (45), the fact that $G \in \mathcal{G}$, and (47), respectively. Thus,

$$
\begin{aligned}
H\left(Y_{2} \mid U\right) & =\sum_{u} P(U=u) H\left(Y_{2} \mid U=u\right) \\
& \geq \sum_{u} P(U=u) F\left(H\left(Y_{1} \mid U=u\right)\right) \\
& \geq \sum_{u} P(U=u) \underline{\operatorname{env}} F\left(H\left(Y_{1} \mid U=u\right)\right) \\
& \geq \underline{\operatorname{env}} F\left(\sum_{u} P(U=u) H\left(Y_{1} \mid U=u\right)\right) \\
& =\underline{\operatorname{env}} F\left(H\left(Y_{1} \mid U\right)\right) \\
& =\underline{\operatorname{env}} F(c)
\end{aligned}
$$

where (53) follows from (51), (54) follows from the definition of env, and (55) follows from convexity of env $F(\cdot)$.

Finally, for $\eta \leq c \leq \log \left|\mathcal{Y}_{1}\right|$, we have

$$
\begin{aligned}
T(c) & =I\left(U ; Y_{2}\right) \\
& =H\left(Y_{2}\right)-H\left(Y_{2} \mid U\right) \\
& \leq \tau-\underline{\text { env }} F(c)
\end{aligned}
$$

where (60) follows from (57) and the definition of $\tau$ in (24).

Therefore, we conclude that the single-letter characterization of the capacity region of DDICs satisfying conditions $1-5$ is (41), and also (18). To achieve point $\left(R_{1}, R_{2}\right)$ on the boundary of the capacity region, if $R_{1}$ and $R_{2}$ are such that

$$
R_{1}=c-\eta, \quad R_{2}=\tau-F(c)
$$

for some $\eta \leq c \leq \log \left|\mathcal{Y}_{1}\right|$, transmitters 1 and 2 generate random codebooks according to $p^{*}\left(x_{1}\right)$, which is the minimizer of $F\left(R_{1}+\eta\right)$, and $p^{*}\left(x_{2}\right)$, which is the uniform distribution, respectively, and transmit the codewords corresponding to the realizations of their own messages. Receiver 1 performs successive decoding, in the order of message 2 , and then message 1. Receiver 2 decodes its own message treating interference from transmitter 1 as pure noise. To achieve point $\left(R_{1}, R_{2}\right)$ on the capacity region, where $R_{1}$ and $R_{2}$ do not satisfy 61, time-sharing should be used. Furthermore, we note that for these DDICs, encoder cooperation cannot increase the capacity region.

\section{EXAMPLES}

In this section, we will provide three examples of DDICs for which conditions 1-5 are satisfied. The first example is the channel model adopted in [1], for which the capacity region is already known. In the second and third examples, the capacity regions are previously unknown, and using the results of this paper, we are able to determine the capacity regions. 


\section{A. Example 1}

A DADIC is defined as [1]

$$
\begin{aligned}
& Y_{1}=X_{1} \oplus X_{2} \oplus V_{1} \\
& Y_{2}=X_{1} \oplus X_{2} \oplus V_{1} \oplus V_{2}
\end{aligned}
$$

where

$$
\mathcal{X}_{1}=\mathcal{X}_{2}=\mathcal{Y}_{1}=\mathcal{Y}_{2}=\mathcal{S}=\{0,1, \cdots, s-1\}
$$

and $\oplus$ denotes modulo- $s$ sum, and $V_{1}$ and $V_{2}$ are independent noise random variables defined over $\mathcal{S}$ with distributions

$$
\mathbf{p}_{i}=\left(p_{i}(0), p_{i}(1), \cdots, p_{i}(s-1)\right), \quad i=1,2
$$

Since $Y_{2}=Y_{1} \oplus V_{2}$, matrix $T^{\prime}$ is circulant, and thus input symmetric [10, Section II.D]. Hence, condition 1 is satisfied. It is straightforward to check that conditions 2-5 are also satisfied. For example, when $s=3$, we have

$$
T^{\prime}=\left[\begin{array}{lll}
p_{2}(0) & p_{2}(2) & p_{2}(1) \\
p_{2}(1) & p_{2}(0) & p_{2}(2) \\
p_{2}(2) & p_{2}(1) & p_{2}(0)
\end{array}\right]
$$

and the input symmetry group for $T^{\prime}$ is

$$
\begin{aligned}
\mathcal{G}=\left\{G_{0}=\left[\begin{array}{lll}
1 & 0 & 0 \\
0 & 1 & 0 \\
0 & 0 & 1
\end{array}\right],\right. & G_{1}=\left[\begin{array}{lll}
0 & 0 & 1 \\
1 & 0 & 0 \\
0 & 1 & 0
\end{array}\right], \\
G_{2} & \left.=\left[\begin{array}{lll}
0 & 1 & 0 \\
0 & 0 & 1 \\
1 & 0 & 0
\end{array}\right]\right\}
\end{aligned}
$$

which is transitive, i.e., $1 \stackrel{G_{2}}{\longrightarrow} 2,1 \stackrel{G_{1}}{\longrightarrow} 3,2 \stackrel{G_{1}}{\longrightarrow} 1,2 \stackrel{G_{2}}{\longrightarrow}$ $3,3 \stackrel{G_{2}}{\longrightarrow} 1,3 \stackrel{G_{1}}{\longrightarrow} 2$. From [62, we write

$$
\begin{aligned}
& T_{0}=\left[\begin{array}{lll}
p_{1}(0) & p_{1}(2) & p_{1}(1) \\
p_{1}(1) & p_{1}(0) & p_{1}(2) \\
p_{1}(2) & p_{1}(1) & p_{1}(0)
\end{array}\right] \\
& T_{1}=\left[\begin{array}{lll}
p_{1}(2) & p_{1}(1) & p_{1}(0) \\
p_{1}(0) & p_{1}(2) & p_{1}(1) \\
p_{1}(1) & p_{1}(0) & p_{1}(2)
\end{array}\right] \\
& T_{2}=\left[\begin{array}{lll}
p_{1}(1) & p_{1}(0) & p_{1}(2) \\
p_{1}(2) & p_{1}(1) & p_{1}(0) \\
p_{1}(0) & p_{1}(2) & p_{1}(1)
\end{array}\right]
\end{aligned}
$$

Conditions 2-4 are satisfied because

$$
\begin{aligned}
T_{1}=G_{1} T_{0}, & T_{2}=G_{2} T_{0} \\
\eta & =H\left(V_{1}\right) \\
\sum_{x_{2}} p\left(y_{1} \mid x_{1}, x_{2}\right) & =p_{1}(0)+p_{1}(1)+p_{1}(2)=1
\end{aligned}
$$

Next, we check condition 5 .

$$
\begin{gathered}
\left\{\sum_{x_{1}, x_{2}} a_{x_{1}, x_{2}} \mathbf{p}_{x_{1}, x_{2}}: \sum_{x_{1}, x_{2}} a_{x_{1}, x_{2}}=1, a_{x_{1}, x_{2}} \geq 0\right\} \\
=\left\{a\left(\begin{array}{l}
p_{1}(0) \\
p_{1}(1) \\
p_{1}(2)
\end{array}\right)+b\left(\begin{array}{l}
p_{1}(2) \\
p_{1}(0) \\
p_{1}(1)
\end{array}\right)+c\left(\begin{array}{l}
p_{1}(1) \\
p_{1}(2) \\
p_{1}(0)
\end{array}\right):\right. \\
a+b+c=1, a, b, c \geq 0\}
\end{gathered}
$$

because even though (74) is a convex combination of 9 vectors, due to vectors repeating themselves in the columns of $T_{0}, T_{1}$ and $T_{2}$, the set, in fact, consists of convex combinations of only 3 vectors. On the other hand, for $\tilde{x}_{2}=0$,

$$
\begin{gathered}
\left\{G\left(\sum_{x_{1}} b_{x_{1}} \mathbf{p}_{x_{1}, \tilde{x}_{2}}\right): \sum_{x_{1}} b_{x_{1}}=1, b_{x_{1}} \geq 0, G=G_{0}\right\} \\
=\left\{a\left(\begin{array}{l}
p_{1}(0) \\
p_{1}(1) \\
p_{1}(2)
\end{array}\right)+b\left(\begin{array}{l}
p_{1}(2) \\
p_{1}(0) \\
p_{1}(1)
\end{array}\right)+c\left(\begin{array}{l}
p_{1}(1) \\
p_{1}(2) \\
p_{1}(0)
\end{array}\right):\right. \\
a+b+c=1, a, b, c \geq 0\}
\end{gathered}
$$

because 76 is the convex combinations of the columns of $T_{0}$, with the unitary permutation. Thus,

$$
\begin{aligned}
& \left\{\sum_{x_{1}, x_{2}} a_{x_{1}, x_{2}} \mathbf{p}_{x_{1}, x_{2}}: \sum_{x_{1}, x_{2}} a_{x_{1}, x_{2}}=1, a_{x_{1}, x_{2}} \geq 0\right\} \\
& =\left\{G\left(\sum_{x_{1}} b_{x_{1}} \mathbf{p}_{x_{1}, \tilde{x}_{2}}\right): \sum_{x_{1}} b_{x_{1}}=1, b_{x_{1}} \geq 0, G=G_{0}\right\} \\
& \subseteq\left\{G\left(\sum_{x_{1}} b_{x_{1}} \mathbf{p}_{x_{1}, \tilde{x}_{2}}\right): \sum_{x_{1}} b_{x_{1}}=1, b_{x_{1}} \geq 0, G \in \mathcal{G}\right\}
\end{aligned}
$$

and condition 5 is satisfied.

\section{B. Example 2}

Next, we consider the following DDIC. We have $\left|\mathcal{X}_{1}\right|=$ $\left|\mathcal{X}_{2}\right|=\left|\mathcal{Y}_{1}\right|=2,\left|\mathcal{Y}_{2}\right|=3$, and $p\left(y_{1} \mid x_{1}, x_{2}\right)$ is characterized by

$$
Y_{1}=X_{1} \oplus X_{2} \oplus V_{1}
$$

where $V_{1}$ is Bernoulli with $p . p^{\prime}\left(y_{2} \mid y_{1}\right)$ is an erasure channel with parameter $0 \leq \alpha \leq 1$, i.e., the transition probability matrix is

$$
T^{\prime}=\left[\begin{array}{cc}
1-\alpha & 0 \\
\alpha & \alpha \\
0 & 1-\alpha
\end{array}\right]
$$

Thus, the channel is such that the "bad" receiver cannot receive all the bits that the "good" receiver receives. More specifically, $\alpha$ proportion of the time, whether the bit is a 0 or 1 is unrecognizable, and thus denoted as an erasure $e$.

It is easy to see that $T^{\prime}$ is input symmetric because the input symmetry group

$$
\mathcal{G}=\left\{\left[\begin{array}{ll}
1 & 0 \\
0 & 1
\end{array}\right],\left[\begin{array}{ll}
0 & 1 \\
1 & 0
\end{array}\right]\right\}
$$

is transitive. Conditions 2-5 are satisfied because $p\left(y_{1} \mid x_{1}, x_{2}\right)$ is the same as in Example 1 in Section $\mathrm{VI}-\mathrm{A}$ 


\section{Example 3}

Let $a, b, c, d, e, f$ be non-negative numbers such that $a+$ $b+c=1$ and $d+e+f=1 / 2$. We have $\left|\mathcal{X}_{1}\right|=4,\left|\mathcal{X}_{2}\right|=$ $\left|\mathcal{Y}_{1}\right|=3$, and $\left|\mathcal{Y}_{2}\right|=6$. The DDIC is described as

$$
\begin{aligned}
T^{\prime} & =\left[\begin{array}{lll}
d & e & f \\
e & f & d \\
d & f & e \\
f & e & d \\
e & d & f \\
f & d & e
\end{array}\right] \\
T_{0} & =\left[\begin{array}{llll}
a & b & c & c \\
b & c & a & b \\
c & a & b & a
\end{array}\right] \\
T_{1} & =\left[\begin{array}{llll}
c & a & b & a \\
a & b & c & c \\
b & c & a & b
\end{array}\right] \\
T_{2} & =\left[\begin{array}{llll}
b & c & a & b \\
c & a & b & a \\
a & b & c & c
\end{array}\right]
\end{aligned}
$$

It is straightforward to see that $T^{\prime}$ is input symmetric because the input symmetry group

$$
\begin{aligned}
\mathcal{G}=\left\{G_{0}\right. & =\left[\begin{array}{lll}
1 & 0 & 0 \\
0 & 1 & 0 \\
0 & 0 & 1
\end{array}\right], G_{1}=\left[\begin{array}{lll}
0 & 0 & 1 \\
1 & 0 & 0 \\
0 & 1 & 0
\end{array}\right], \\
G_{2} & =\left[\begin{array}{lll}
0 & 1 & 0 \\
0 & 0 & 1 \\
1 & 0 & 0
\end{array}\right], G_{3}=\left[\begin{array}{lll}
1 & 0 & 0 \\
0 & 0 & 1 \\
0 & 1 & 0
\end{array}\right], \\
G_{4} & \left.=\left[\begin{array}{lll}
0 & 1 & 0 \\
1 & 0 & 0 \\
0 & 0 & 1
\end{array}\right], G_{5}=\left[\begin{array}{lll}
0 & 0 & 1 \\
0 & 1 & 0 \\
1 & 0 & 0
\end{array}\right]\right\}
\end{aligned}
$$

is transitive. Conditions 2-4 are satisfied because

$$
\begin{gathered}
T_{1}=G_{1} T_{0}, \quad T_{2}=G_{2} T_{0} \\
\eta=-a \log a-b \log b-c \log c \\
\sum_{x_{2}} p\left(y_{1} \mid x_{1}, x_{2}\right)=a+b+c=1
\end{gathered}
$$

To show condition 5, we use Figure 11 The set on the first line of (6) in condition 5 is the convex combination of the following six points,

$$
\left[\begin{array}{l}
a \\
b \\
c
\end{array}\right],\left[\begin{array}{l}
a \\
c \\
b
\end{array}\right],\left[\begin{array}{l}
c \\
a \\
b
\end{array}\right],\left[\begin{array}{l}
b \\
a \\
c
\end{array}\right],\left[\begin{array}{l}
b \\
c \\
a
\end{array}\right],\left[\begin{array}{l}
c \\
b \\
a
\end{array}\right]
$$

resulting in all the points within the hexagon in Figure 1 The three sets

$$
\begin{aligned}
& \left\{G\left(\sum_{x_{1}} b_{x_{1}} \mathbf{p}_{x_{1}, \tilde{x}_{2}}\right): \sum_{x_{1}} b_{x_{1}}=1, b_{x_{1}} \geq 0, G=G_{0}\right\} \\
= & \left\{\mu_{1}\left[\begin{array}{l}
a \\
b \\
c
\end{array}\right]+\mu_{2}\left[\begin{array}{l}
b \\
c \\
a
\end{array}\right]+\mu_{3}\left[\begin{array}{l}
c \\
a \\
b
\end{array}\right]+\mu_{4}\left[\begin{array}{l}
c \\
b \\
a
\end{array}\right]:\right. \\
& \left.\sum_{i=1}^{4} \mu_{i}=1, \mu_{i} \geq 0\right\}
\end{aligned}
$$

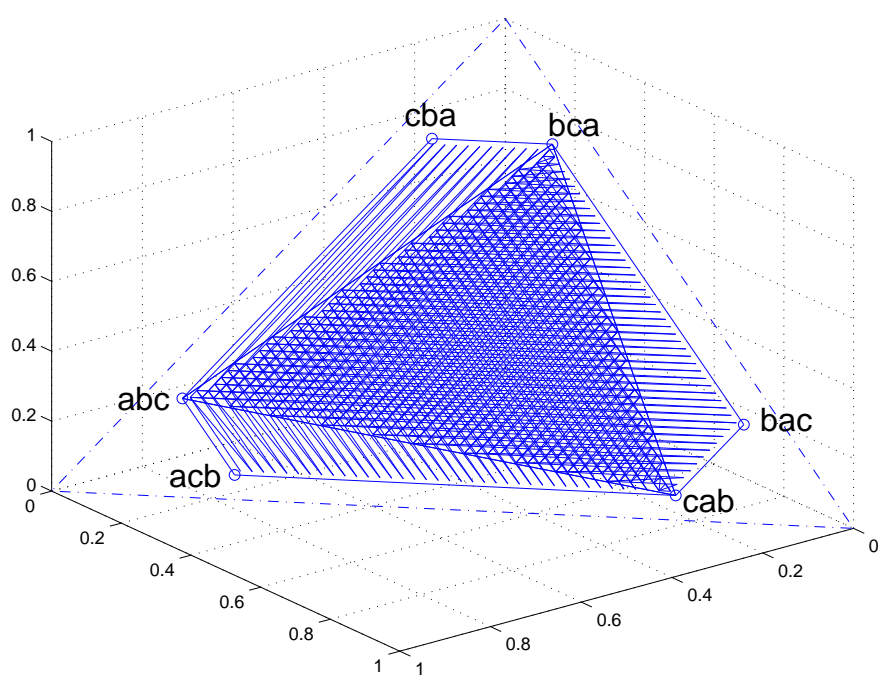

Fig. 1. Explanation of condition 5 in example 3.

and

$$
\begin{aligned}
&\left\{G\left(\sum_{x_{1}} b_{x_{1}} \mathbf{p}_{x_{1}, \tilde{x}_{2}}\right): \sum_{x_{1}} b_{x_{1}}=1, b_{x_{1}} \geq 0, G=G_{1}\right\} \\
&=\left\{\mu_{1}\left[\begin{array}{l}
c \\
a \\
b
\end{array}\right]+\mu_{2}\left[\begin{array}{l}
a \\
b \\
c
\end{array}\right]+\mu_{3}\left[\begin{array}{l}
b \\
c \\
a
\end{array}\right]+\mu_{4}\left[\begin{array}{l}
a \\
c \\
b
\end{array}\right]:\right. \\
&\left.\sum_{i=1}^{4} \mu_{i}=1, \mu_{i} \geq 0\right\}
\end{aligned}
$$

and

$$
\begin{gathered}
\left\{G\left(\sum_{x_{1}} b_{x_{1}} \mathbf{p}_{x_{1}, \tilde{x}_{2}}\right): \sum_{x_{1}} b_{x_{1}}=1, b_{x_{1}} \geq 0, G=G_{2}\right\} \\
=\left\{\mu_{1}\left[\begin{array}{l}
b \\
c \\
a
\end{array}\right]+\mu_{2}\left[\begin{array}{l}
c \\
a \\
b
\end{array}\right]+\mu_{3}\left[\begin{array}{l}
a \\
b \\
c
\end{array}\right]+\mu_{4}\left[\begin{array}{l}
b \\
a \\
c
\end{array}\right]:\right. \\
\left.\sum_{i=1}^{4} \mu_{i}=1, \mu_{i} \geq 0\right\}
\end{gathered}
$$

correspond to the points in the three shaded areas, [abc, $c b a, b c a, c a b],[a c b, a b c, b c a, c a b]$, and $[b a c, c a b, a b c, b c a]$, respectively. Since the three shaded areas cover the entire hexagon, and $\left\{G_{0}, G_{1}, G_{2}\right\} \subset \mathcal{G}$, condition 5 is satisfied.

\section{CONCLUSION}

We provide a single-letter characterization for the capacity region of a class of DDICs, which is more general than the class of DADICs studied by Benzel [1]. We show that for the class of DDICs studied, encoder cooperation does not increase the capacity region, and the best way to manage the interference is through random codebook design and treating the signal for the "good" receiver as pure noise at the "bad" receiver. 


\section{REFERENCES}

[1] R. Benzel, "The capacity region of a class of discrete additive degraded interference channels," IEEE Trans. on Information Theory, vol. 25, pp. 228-231, March 1979.

[2] C. E. Shannon, "Two-way communication channels," Proc. 4th Berkeley Symp. Math. Stat. Prob., pp. 611-644, 1961.

[3] A. El Gamal and M. Zahedi, "The capacity region of a class of deterministic interference channels," IEEE Trans. on Information Theory, vol. 28, no. 2, pp. 343-346, March 1982.

[4] H. Sato, "The capacity of the Gaussian interference channel under strong interference," IEEE Trans. on Information Theory, vol. 27, pp. 786-788, November 1981.

[5] M. Costa and A. El Gamal, "The capacity region of the discrete memoryless interference channel with strong interference," IEEE Trans. on Information Theory, vol. 33, no. 5, pp. 710-711, September 1987.

[6] A. B. Carleial, "Interference channels," IEEE Trans. on Information Theory, vol. 24, pp. 60-70, January 1978.

[7] H. Sato, "The two-user communication channels," IEEE Trans. on Information Theory, vol. 23, pp. 295-304, May 1977.

[8] R. Ahlswede, "Multi-way communication channels," in Proc. 2nd Int. Symp. Inform. Theory, Tsahkadsor, Armenian S.S.R., 1971.

[9] T. M. Cover and J. A. Thomas, Elements of Information Theory. Wiley-Interscience, 1991.

[10] H. Witsenhausen and A. Wyner, "A conditional entropy bound for a pair of discrete random variables," IEEE Trans. on Information Theory, vol. 21, no. 5, pp. 493-501, September 1975.

[11] T. M. Cover, "Broadcast channels," IEEE Trans. on Information Theory, vol. 18, no. 1, pp. 2-14, January 1972.

[12] R. G. Gallager, "Capacity and coding for degraded broadcast channels," Problemy Peredaci Informaccii, vol. 10, no. 3, pp. 3-14, 1974.

[13] R. Ahlswede and J. Korner, "Source coding with side information and a converse for degraded broadcast channels," IEEE Trans. on Information Theory, vol. 21, pp. 629 - 637, November 1975.

[14] H. Witsenhausen, "Entropy inequalities for discrete channels," IEEE Trans. on Information Theory, vol. 20, no. 5, pp. 610-616, September 1974. 